\title{
Treatment of $\gamma_{5}$ in Dimensionally-Regularized Chiral Yang-Mills Theory with Scalar Fields
}

\author{
Hermès Bélusca-Maïto* \\ Department of Physics, Faculty of Sciences, University of Zagreb, \\ Bijenička cesta 32, HR-10000 Zagreb, Croatia \\ E-mail: hbelusca@phy.hr

\begin{abstract}
Amon llakovac, Marija Mađor-Božinović
Department of Physics, Faculty of Sciences, University of Zagreb,

Bijenička cesta 32, HR-10000 Zagreb, Croatia

E-mail: ailakov@phy.hr, mmadjor@phy.hr
\end{abstract}

\section{Dominik Stöckinger}

Institut für Kern- und Teilchenphysik, TU Dresden,

DE-01069 Dresden, Germany

E-mail: Dominik.Stoeckinger@tu-dresden.de

\begin{abstract}
We present in these proceedings, our analysis [1] at one-loop level within the BreitenlohnerMaison-'t Hooft-Veltman (BMHV) scheme of Dimensional Regularization, of the counterterm structure arising due to the presence of the $\gamma_{5}$ matrix and the breaking of the BRST invariance, in a massless chiral Yang-Mills theory supplemented with real scalars. The full one-loop counterterm structure necessary for the renormalization is obtained. This comprises the singular counterterms, including evanescent ones, and by using techniques from algebraic renormalization the symmetry-restoring finite counterterms are evaluated as well. The latter are required to restore the BRST invariance, central to the consistency of the theory and for higher-order calculations. The renormalization group equations are derived within this framework, and are compared with the more "familiar" calculation in the context of symmetry-invariant renormalization.
\end{abstract}

Preprint number: ZTF-EP-20-02

PACS: 03.65.Fd, 11.10.-z, 11.10.Gh, 11.10.Hi, 11.15.-q, 11.30.Rd

Corfu Summer Institute 2019 "School and Workshops on Elementary Particle Physics and Gravity"

(CORFU2019)

31 August - 25 September 2019

Corfu, Greece

${ }^{*}$ Speaker. 


\section{Introduction}

Dimensional Regularization (DReg) [2, 3, 4, 5] is one of the most employed schemes for regularizing, while explicitly preserving Lorentz covariance, the divergences arising from loop calculations in quantum field theories (QFTs) into poles in a dimension-shift parameter $v$. Taking the case of 4-dimensional QFTs extended to a formal " $d$ "-dimensional space, $v$ is usually equal to $4-d$. The $d$-dimensional space is separated into a direct sum of 4 and $d-4 \equiv-2 \varepsilon$ dimensional subspaces. All Lorentz-covariant objects present are extended from 4 to $d$ dimensions, and acquire 4-dimensional (denoted by bars: ${ }^{-}$) and $(-2 \varepsilon)$-dimensional "evanescent" (denoted by hats: $\widehat{\cdot}$ ) components. On these subspaces, the metric tensors (also understood as projectors) are defined by

$$
d \text {-dim. : } g_{\mu v}, \quad \text { 4-dim. }: \bar{g}_{\mu \nu}, \quad(-2 \varepsilon) \text {-dim. }: \hat{g}_{\mu \nu}=g_{\mu v}-\bar{g}_{\mu v},
$$

and similar for their inverse (with upper indices, see Ref. [6]); using a mostly minus signature $(+1,-1,-1,-1)$ for the 4-dimensional metric, their contractions are

$$
g_{\mu \nu} g^{v \mu}=d, \quad \bar{g}_{\mu \nu} \bar{g}^{v \mu}=4, \quad \hat{g}_{\mu v} \hat{g}^{v \mu}=d-4 \equiv-2 \varepsilon,
$$

and

$$
\begin{array}{rlrl}
g_{\mu \nu} g^{v \rho} & =g_{\mu}^{\rho} \equiv \delta_{\mu}^{\rho}, & \bar{g}_{\mu \nu} \bar{g}^{v \rho}=\bar{g}_{\mu}^{\rho}=\bar{g}_{\mu \nu} g^{v \rho}=g_{\mu \nu} \bar{g}^{v \rho}, \\
\hat{g}_{\mu \nu} \hat{g}^{v \rho}=\hat{g}_{\mu}^{\rho}=\hat{g}_{\mu \nu} g^{v \rho}=g_{\mu \nu} \hat{g}^{v \rho}, & \bar{g}_{\mu \nu} \hat{g}^{v \rho}=0=\hat{g}_{\mu \nu} \bar{g}^{v \rho} .
\end{array}
$$

$\gamma^{\mu}$ Dirac matrices can be extended as well to $d$ dimensions (see e.g. Chapter 4 of [6]), with 4-dimensional $\bar{\gamma}^{\mu}$ and $(-2 \varepsilon)$-dimensional $\hat{\gamma}^{\mu}$ components. (Anti-)commutation and contraction properties for these objects can be obtained as well (see e.g. in [1]).

The description of chiral fermions in 4-dimensional QFTs, leads to the appearance of the $\gamma_{5}$ matrix, or related, of the Levi-Civita symbol $\varepsilon_{\mu v \rho \sigma}$. Thus arises the question of how to define these intrinsically 4-dimensional quantities in DReg. Naively manipulating these objects would lead to inconsistencies, so one should instead find a way to ensure that the Ward or the Slavnov-Taylor identities are satisfied, either by restoring them or by ensuring their validity without any restoration.

In these proceedings we summarize our study of this problem [1], made in the context of the renormalization of a $d$-dimensional massless chiral gauge theory (Section 2). We adopt the socalled BMHV scheme for handling $\gamma_{5}$ and $\varepsilon_{\mu v \rho \sigma}$, formalized by 't Hooft and Veltman $[5,7,8,9]$, proven to be axiomatically consistent perturbatively at all loop orders by Breitenlohner and Maison $[10,11,12,13]$, and being able to reproduce the ABJ anomaly [14]. In this scheme, the $\varepsilon$ symbol is defined by its product with the metric tensor, and with itself, (using the $\varepsilon^{0123}=+1$ convention)

$$
g_{\mu}^{\mu_{1}} \varepsilon_{\mu_{1} \mu_{2} \mu_{3} \mu_{4}}=\varepsilon_{\mu \mu_{2} \mu_{3} \mu_{4}}, \quad \varepsilon_{\mu_{1} \mu_{2} \mu_{3} \mu_{4}} \varepsilon_{v_{1} v_{2} v_{3} v_{4}}=-\sum_{\pi \in S_{4}} \operatorname{sgn}(\pi) \prod_{i=1}^{4} \bar{g}_{\mu_{i} v_{\pi(i)}}
$$

while $\gamma_{5}$ possesses both anticommuting and commuting properties:

$$
\left\{\gamma_{5}, \bar{\gamma}^{\mu}\right\}=0, \quad\left[\gamma_{5}, \hat{\gamma}^{\mu}\right]=0, \quad\left\{\gamma_{5}, \gamma^{\mu}\right\}=\left\{\gamma_{5}, \hat{\gamma}^{\mu}\right\}=2 \gamma_{5} \hat{\gamma}^{\mu}, \quad\left[\gamma_{5}, \gamma^{\mu}\right]=\left[\gamma_{5}, \bar{\gamma}^{\mu}\right]=2 \gamma_{5} \bar{\gamma}^{\mu}
$$

$\gamma_{5}$ otherwise keeps its usual 4-dimensional behaviour, from its explicit definition [10]:

$$
\gamma_{5}=\frac{-i}{4 !} \varepsilon_{\mu v \rho \sigma} \gamma^{\mu} \gamma^{v} \gamma^{\rho} \gamma^{\sigma}, \quad \gamma_{5}^{2}=\mathbb{1}
$$


We analyse (Section 4) the counterterm structure arising in this scheme and the induced breaking of the BRST invariance (Section 3). For the consistence of the theory, this symmetry is restored via suitable finite BRST-restoring counterterms (Section 5), obtained via the framework of algebraic renormalization.

Besides, the chiral fermions generate chiral anomalies $[15,16]$ manifested e.g. in pion decays in the Standard Model (SM), formally arising from the presence of $\gamma_{5}$ in a fermion trace (the AdlerBell-Jackiw (ABJ) anomaly),

$$
\operatorname{Tr}\left(\left\{\gamma^{\alpha}, \gamma_{5}\right\} \gamma_{\alpha} \gamma_{\mu} \gamma_{\nu} \gamma_{\rho} \gamma_{\sigma}\right)=8 i(d-4) \varepsilon_{\mu v \rho \sigma}
$$

Gauge theories are well-defined and renormalizable only if chiral gauge anomalies are absent (the one-loop anomaly cancellation conditions from the Adler-Bardeen theorem [17]). Placing ourselves in this case, we then evaluate renormalization group equations at one-loop level (Section 6) using the algebraic method. They are compared with the more customary approach from renormalization transformations, and we see why no differences arise between the two approaches at one-loop. However, possible changes can be anticipated in two- or higher loops calculations.

\section{Chiral Right-Handed Model in $d$ Dimensions}

We employ a massless chiral gauge theory, similar to the one from Refs. [18, 19, 20], based on a simple gauge Lie group $\mathscr{G}$ with gauge fields $G_{\mu}^{a}$ (structure constants $f^{a b c}$ ). The matter fields, charged under $\mathscr{G}$ and having irreducible representations for simplicity, are massless right-handed fermions (rep. 'R', generators $T_{R i j}^{a}$ ), and real massless scalars $\Phi^{m}$ (rep. 'S', generators $\theta_{m n}^{a}$ ).

The massless right-handed fermion fields are described with projections of Dirac spinors: $\mathbb{P}_{\mathrm{R}} \psi \equiv \psi_{R}$, since these can be generalized to $d$ dimensions. The left-handed part of $\psi$ is fictitious, decoupled from the theory. We use the standard right/left chirality projectors $\mathbb{P}_{\mathrm{R}, \mathrm{L}}=\left(\mathbb{1} \pm \gamma_{5}\right) / 2$. Thus, the fermionic Lagrangian density in 4 dimensions can be written as (using $\overline{\psi_{R}}=\bar{\psi} \mathbb{P}_{\mathrm{L}}$ ):

$$
\mathscr{L}_{\text {fermions }}=i \overline{\psi_{R}} \not D^{i j} \psi_{R_{j}}=i \overline{\psi_{R}} \not \partial \psi_{R_{i}}+g T_{R i j}^{a} \overline{\psi_{R}} \phi^{a} \psi_{R_{j}}
$$

The left-handed part $\mathbb{P}_{L} \psi$ entirely decouples and does not appear at all in this Lagrangian. The other usual Lagrangian densities (gauge, scalar and Yukawa terms) are

$$
\begin{gathered}
\mathscr{L}_{\text {gauge }}=\frac{-1}{4} F_{\mu v}^{a} F^{a \mu v}, \quad \mathscr{L}_{\text {scalars }}=\frac{1}{2}\left(D_{\mu} \Phi^{m}\right)^{2}-\frac{\lambda^{m n o p}}{4 !} \Phi_{m} \Phi_{n} \Phi_{o} \Phi_{p}, \\
\mathscr{L}_{\text {Yukawa }}=-\frac{\left(Y_{R}\right)_{i j}^{m}}{2} \Phi_{m}{\overline{\psi_{R}}}_{i}^{C} \psi_{R_{j}}-\frac{\left(Y_{R}\right)_{i j}^{m *}}{2} \Phi_{m} \overline{\psi_{R}} \psi_{R j}^{C} .
\end{gathered}
$$

The covariant derivative (with the generator $X_{i j}^{a} \equiv T_{R}^{a}$ for fermions, and $X_{i j}^{a} \equiv \theta_{m n}^{a}$ for scalars), and the field-strength tensor for $G$, are defined as:

$$
\begin{gathered}
D_{i j \mu}=\partial_{\mu} \delta_{i j}-i g G_{\mu}^{a} X_{i j}^{a}, \\
F_{\mu \nu}^{a}=\partial_{\mu} G_{v}^{a}-\partial_{\nu} G_{\mu}^{a}+g f^{a b c} G_{\mu}^{b} G_{\nu}^{c} .
\end{gathered}
$$

The Yukawa matrix $\left(Y_{R}\right)_{i j}^{m}$ is symmetric in its fermion-group indices $i, j$. 
Note that the Yukawa interactions contains charge-conjugated Dirac fermions. It is indeed possible to define the charge-conjugation operation $\widehat{\mathscr{C}}$ in continuous $d \approx 4$ dimensions in the dimensional regularization scheme. A corresponding matrix representation $C$ can explicitly be constructed, see e.g. Appendix A of [21]. Alternatively, $\widehat{\mathscr{C}}$ can be defined only from its action on the set of Dirac matrices and on the $d$-dimensional spinors. Since we work in dimension $d=4-2 \varepsilon$ around 4 , we postulate that the charge conjugation properties that hold in 4 dimensions, viz.

$$
C^{-1}=C^{\dagger}=C^{T}, \quad C^{T}=-C,
$$

using the matrix representation, also hold in $d \approx 4$ (motivated e.g. in Appendix A of [22]), together with the following properties and action on anticommuting fermions (Appendix G.1 of [23]):

$$
\begin{gathered}
C^{-1} \Gamma C=\eta_{\Gamma} \Gamma^{T} \Rightarrow C \Gamma^{T} C^{-1}=\eta_{\Gamma} \Gamma, \text { with: } \eta_{\Gamma}= \begin{cases}+1 & \text { for } \Gamma=\mathbb{1}, \gamma_{5}, \\
-1 & \text { for } \Gamma=\gamma^{\mu}, \sigma^{\mu v},\end{cases} \\
\widehat{\mathscr{C}} \Psi \widehat{\mathscr{C}}^{-1} \equiv \Psi^{C}=C \bar{\Psi}^{T}, \quad\left(\Psi^{C}\right)^{C}=\Psi, \quad \widehat{\mathscr{C}} \Psi_{\mathscr{C}^{-1}} \equiv \Psi^{C}=-\Psi^{T} C^{-1}=\overline{\Psi^{C}}, \\
\bar{\Psi}_{i}^{C} \Gamma \Psi_{j}^{C}=-\Psi_{i}^{T} C^{-1} \Gamma C \bar{\Psi}_{j}^{T}=\bar{\Psi}_{j} C \Gamma^{T} C^{-1} \Psi_{i}=\eta_{\Gamma} \bar{\Psi}_{j} \Gamma \Psi_{i} .
\end{gathered}
$$

This ensures that the Yukawa term, and any other quantity involving these charge-conjugated fermions, will be meaningful in $d$ dimensions.

All these Lagrangian densities are straightforwardly extended to $d$ dimensions, excepted for $\mathscr{L}_{\text {fermions. }}$ The first difficulty concerns the fermion-gauge interaction term in Eq. (2.1), which involves the 4-dimensional right-handed current $\bar{\psi}_{i} \gamma^{\mu} \psi_{R_{j}}$. Because $\mathbb{P}_{\mathrm{L}} \gamma^{\mu} \neq \gamma^{\mu} \mathbb{P}_{\mathrm{R}}$ in $d$ dimensions, see Eq. (1.4), three inequivalent choices for the $d$-dimensional versions of this term are possible:

$$
\bar{\psi}_{i} \gamma^{\mu} \mathbb{P}_{\mathrm{R}} \psi_{j}, \quad \bar{\psi}_{i} \mathbb{P}_{\mathrm{L}} \gamma^{\mu} \psi_{j}, \quad \bar{\psi}_{i} \mathbb{P}_{\mathrm{L}} \gamma^{\mu} \mathbb{P}_{\mathrm{R}} \psi_{j}
$$

Each of these generate different, but perfectly valid renormalizable $d$-dimensional extensions of the model in dimensional regularization and the BMHV scheme. Here we choose the third option,

$$
\bar{\psi} \mathbb{P}_{\mathrm{L}} \gamma^{\mu} \mathbb{P}_{\mathrm{R}} \psi=\bar{\psi} \mathbb{P}_{\mathrm{L}} \bar{\gamma}^{\mu} \mathbb{P}_{\mathrm{R}} \psi=\bar{\psi}_{R} \gamma^{\mu} \psi_{R}
$$

as it leads to the simplest expressions, and carries the information that right-handed fermions were originally present on both sides of the interaction term. The second problem, is that the fermionic kinetic term $i \bar{\psi}_{R_{i}} \partial \psi_{R_{i}}=i \bar{\psi}_{i} \mathbb{P}_{\mathrm{L}} \partial \mathbb{P}_{\mathrm{R}} \psi_{i}$ generates only a purely 4-dimensional propagator and leads to unregularized loop diagrams. The solution is to use instead the entire Dirac fermion $\psi$ and the fully $d$ dimensional covariant kinetic term $i \bar{\psi}_{i} \not \partial \psi_{i}$. The fictitious left-chiral field $\psi_{L}$ is thus introduced, but appears only within the kinetic term, has no couplings, and is a gauge singlet.

Hence, our dimensionally regularized fermionic kinetic and gauge interaction terms are:

$$
\begin{aligned}
\mathscr{L}_{\text {fermions }} & =i \bar{\psi}_{i} \not \partial \psi_{i}+g T_{R_{i j}}^{a} \overline{\psi_{R}}{ }_{i}{ }^{a} \psi_{R_{j}}=\mathscr{L}_{\text {fermions,inv }}+\mathscr{L}_{\text {fermions,evan }}, \\
\mathscr{L}_{\text {fermions,inv }} & =i \bar{\psi}_{i} \bar{\partial} \psi_{i}+g T_{R i j}{ }^{a} \overline{\psi_{R}}{ }_{i}{ }^{a} \psi_{R_{j}}, \\
\mathscr{L}_{\text {fermions,evan }} & =i \bar{\psi}_{i} \widehat{\partial} \psi_{i}=i \bar{\psi}_{L_{i}} \widehat{\partial} \psi_{R_{i}}+i \bar{\psi}_{R} \hat{\partial} \psi_{L_{i}},
\end{aligned}
$$

$\mathscr{L}_{\text {fermions,inv }}$ contains purely 4-dimensional derivatives and gauge fields. It is gauge and BRST-

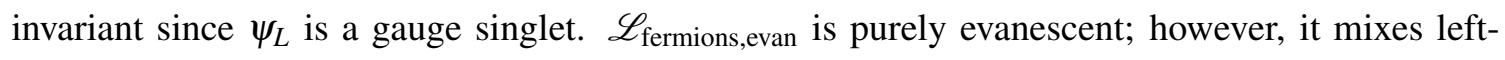
and right-chiral fields that have different gauge transformations, causing the breaking of gauge and BRST invariance, see Section 3. 


\section{Gauge-fixing and quantization in the BRST framework}

The gauge symmetry is promoted to the BRST symmetry and a Slavnov-Taylor identity [24, 25], and the $d$-dimensional BRST transformations of the ordinary fields are formally defined exactly as they would be in 4 dimensions [1]:

$$
\begin{aligned}
s_{d} G_{\mu}^{a} & =D_{\mu}^{a b} c^{b}=\partial_{\mu} c^{a}+g f^{a b c} G_{\mu}^{b} c^{c}, \\
s_{d} \psi_{i} & =s_{d} \psi_{R_{i}}=i c^{a} g T_{R_{i j}}^{a} \psi_{R_{j}}, \\
s_{d} \bar{\psi}_{i} & =s_{d} \overline{\psi_{R}}=+i \overline{\psi_{R}} c^{a} g T_{R j i}^{a}, \\
s_{d} \psi_{L_{i}} & =0, \quad s_{d} \overline{\psi_{L_{i}}}=0, \\
s_{d} \Phi_{m} & =i c^{a} g \theta_{m n}^{a} \Phi_{n} .
\end{aligned}
$$

$c^{a}$ is the Faddeev-Popov ghost field (in the adjoint representation), and $s_{d}$ is the generator of the BRST transformation, acting as a nilpotent fermionic differential operator. The transformations of the ghost $c^{a}$, the antighost $\bar{c}^{a}$ and the auxiliary Nakanishi-Lautrup $B^{a}$ fields are:

$$
s_{d} c^{a}=-\frac{1}{2} g f^{a b c} c^{b} c^{c} \equiv i g c^{2}, \quad s_{d} \bar{c}^{a}=B^{a}, \quad s_{d} B^{a}=0 .
$$

The Lagrangian of the theory is then extended with the ghost and the gauge-fixing terms,

$$
\begin{aligned}
\mathscr{L}_{\text {ghost }} & =\partial^{\mu} \bar{c}_{a} \cdot D_{\mu}^{a b} c_{b} \equiv-\bar{c}_{a} \partial^{\mu} D_{\mu}^{a b} c_{b}, \\
\mathscr{L}_{\text {g-fix }} & =\frac{\xi}{2} B^{a} B_{a}+B^{a} \partial^{\mu} G_{\mu}^{a} .
\end{aligned}
$$

$\mathscr{L}_{\text {g-fix }}$ is equivalent to the more common form: $\mathscr{L}_{\text {g-fix }}=\frac{-1}{2 \xi}\left(\partial^{\mu} G_{\mu}^{a}\right)^{2}$, obtained after integrating out $B^{a}$. Finally, the non-linear BRST transformations are coupled to external sources and the corresponding terms are added to the Lagrangian (see e.g. references in [26]),

$$
\mathscr{L}_{\mathrm{ext}}=\rho_{a}^{\mu} s_{d} G_{\mu}^{a}+\zeta_{a} s_{d} c^{a}+\bar{R}^{i} s_{d} \psi_{R_{i}}+R^{i} s_{d} \overline{\psi_{R}}+\mathscr{Y}^{m} s_{d} \Phi_{m},
$$

where the external sources $\rho_{a}^{\mu}, \zeta_{a}, R, \bar{R}, \mathscr{Y}^{m}$ do not transform under BRST transformations.

\section{The complete $d$-dimensional tree-level action}

The full $d$-dimensional tree-level action $S_{0}$ of the model can be naturally decomposed into a BRST-invariant and a purely evanescent part:

$$
\begin{gathered}
S_{0}=S_{0, \text { inv }}+S_{0, \text { evan }}, \quad \text { with: } \\
S_{0, \text { inv }}=\int \mathrm{d}^{d} x\left(\mathscr{L}_{\text {gauge }}+\mathscr{L}_{\text {fermions,inv }}+\mathscr{L}_{\text {scalars }}+\mathscr{L}_{\text {Yukawa }}+\mathscr{L}_{\text {ghost }}+\mathscr{L}_{\text {g-fix }}+\mathscr{L}_{\text {ext }}\right), \\
S_{0, \text { evan }}=\int \mathrm{d}^{d} x \mathscr{L}_{\text {fermions,evan }} .
\end{gathered}
$$

All terms except $\mathscr{L}_{\text {fermions }}$ are the $d$-dimensional extensions of their corresponding 4-dimensional versions (i.e. all Lorentz indices interpreted in $d$ dimensions). Here, $S_{0, \text { inv }}$ contains everything except the evanescent part of the $d$-dimensional fermion kinetic term Eq. (2.7a). It is clearly BRSTinvariant since the 4-dimensional part of the fermion covariant derivative term is gauge and BRSTinvariant and all other sectors of the theory are insensitive to the transition from 4 to $d$ dimensions. 
The $d$-dimensional tree-level action can also be decomposed as a sum of integrated field mono-

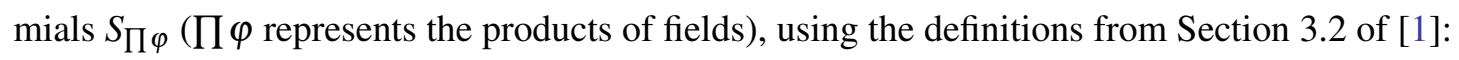

$$
\begin{aligned}
S_{0}= & \left(S_{G G}+S_{G G G}+S_{G G G G}\right)+\left(S_{\bar{\psi} \psi}+\overline{S_{\bar{\psi} G \psi_{R}}}\right)+\left(S_{\Phi \Phi}+S_{\Phi G \Phi}+S_{\Phi G G \Phi}\right) \\
& +\left(\left(Y_{R}\right)_{i j}^{m} S_{{\overline{\psi_{R}}}^{C} \Phi^{m} \psi_{R_{j}}}+\text { h.c. }\right)+\lambda_{\text {mnop }} S_{\Phi_{m n o p}^{4}} \\
& +S_{\text {g-fix }}+\left(S_{\bar{c} c}+S_{\bar{c} G c}\right)+\left(S_{\rho c}+S_{\rho G c}\right)+S_{\zeta c c}+S_{\bar{R} c \psi_{R}}+S_{R c \overline{\psi_{R}}}+S_{\mathscr{Y} c \Phi} .
\end{aligned}
$$

As already announced, this action will break the BRST invariance already at tree-level, see Section 3. However, the projected 4-dimensional tree-level action, given by

$$
S_{0}^{(4 D)}=\int \mathrm{d}^{4} x\left(\mathscr{L}_{\text {gauge }}+\mathscr{L}_{\text {fermions,inv }}+\mathscr{L}_{\text {scalars }}+\mathscr{L}_{\text {Yukawa }}+\mathscr{L}_{\text {ghost }}+\mathscr{L}_{\text {g-fix }}+\mathscr{L}_{\text {ext }}\right),
$$

does not have any evanescent component and is BRST-invariant.

\section{Tree-level BRST breaking}

As mentioned above, $S_{0}^{(4 D)}$ is BRST-invariant, and satisfies the Slavnov-Taylor identity

$$
\mathscr{S}\left(S_{0}^{(4 D)}\right)=0,
$$

where the Slavnov-Taylor operation is given for a general functional $\mathscr{F}$ as

$$
\mathscr{S}(\mathscr{F})=\int \mathrm{d}^{4} x\left(\frac{\delta \mathscr{F}}{\delta \rho_{a}^{\mu}} \frac{\delta \mathscr{F}}{\delta G_{\mu}^{a}}+\frac{\delta \mathscr{F}}{\delta \zeta_{a}} \frac{\delta \mathscr{F}}{\delta c^{a}}+\frac{\delta \mathscr{F}}{\delta \mathscr{Y}^{m}} \frac{\delta \mathscr{F}}{\delta \Phi_{m}}+\frac{\delta \mathscr{F}}{\delta \bar{R}^{i}} \frac{\delta \mathscr{F}}{\delta \psi_{i}}+\frac{\delta \mathscr{F}}{\delta R^{i}} \frac{\delta \mathscr{F}}{\delta \bar{\psi}_{i}}+B^{a} \frac{\delta \mathscr{F}}{\delta \bar{c}_{a}}\right) .
$$

The Slavnov-Taylor identity is the basic, defining symmetry property of the theory. We will require that the Slavnov-Taylor identity $\mathscr{S}(\Gamma)=0$ is satisfied for the fully renormalized, finite effective action $\Gamma$ (which incorporates the tree-level action, loop corrections and counterterm contributions).

The breaking of the BRST symmetry by the $d$-dimensional model is given only by the contribution from the evanescent part $S_{0, \text { evan }}$ of the action $S_{0}$,

$$
s_{d} S_{0}=s_{d} S_{0, \text { evan }} \equiv \widehat{\Delta},
$$

which translates, from the Quantum Action Principle, to a non-zero contribution to the corresponding $d$-dimensional Slavnov-Taylor identity

$$
\mathscr{S}_{d}\left(S_{0}\right)=\widehat{\Delta}
$$

where the Slavnov-Taylor operator $\mathscr{S}_{d}$ is defined in the same way as its 4-dimensional version in Eq. (3.2) except for replacing all 4-dimensional objects by $d$-dimensional ones. The breaking vertex operator evaluates to

$$
\widehat{\Delta}=\int \mathrm{d}^{d} x\left(g T_{R i j}^{a}\right) c^{a}\left\{\bar{\psi}_{i}\left(\overleftarrow{\widehat{\partial}} \mathbb{P}_{\mathrm{R}}+\overrightarrow{\widehat{\partial}} \mathbb{P}_{\mathrm{L}}\right) \psi_{j}\right\} \equiv \int \mathrm{d}^{d} x \widehat{\Delta}(x) .
$$

It is also useful to provide the breaking vertex expressed in terms of charge-conjugated fermions:

$$
\widehat{\Delta}=\int \mathrm{d}^{d} x\left(g T_{\bar{R} i j}^{a}\right) c^{a}\left\{{\overline{\psi^{C}}}_{i}\left(\overleftarrow{\widehat{\partial}} \mathbb{P}_{\mathrm{L}}+\overrightarrow{\widehat{\partial}} \mathbb{P}_{\mathrm{R}}\right) \psi_{j}^{C}\right\},
$$

where the generator $T_{\bar{R}}{ }_{i j}^{a}$ is the one for the fermionic conjugate representation of $R$. 


\section{Generic Counterterm Structure; One-loop Singular Counterterms}

When employing a regularization scheme that assumes the preservation of gauge and BRST invariance, the corresponding counterterm structure of the theory acquires the simple form of additive renormalization transformation for the physical parameters, and multiplicative renormalization for the fields, as shown in Table 1. The fictitious left-chiral fermion field does not renormalize.

\begin{tabular}{|c|c|}
\hline \multicolumn{2}{|c|}{ Couplings } \\
\hline \multicolumn{2}{|c|}{$g \rightarrow g+\delta g$} \\
\hline \multicolumn{2}{|c|}{$\left(Y_{R}\right)_{i j}^{m} \rightarrow\left(Y_{R}\right)_{i j}^{m}+\delta\left(Y_{R}\right)_{i j}^{m}$} \\
\hline \multicolumn{2}{|c|}{$\lambda^{\text {mnop }} \rightarrow \lambda^{\text {mnop }}+\delta \lambda^{\text {mnop }}$} \\
\hline Dynamical fields & BRST Sources \\
\hline$G_{\mu}^{a} \rightarrow \sqrt{Z_{G}} G_{\mu}^{a}$ & $\rho_{a}^{\mu} \rightarrow{\sqrt{Z_{G}}}^{-1} \rho_{a}^{\mu}$ \\
\hline$c^{a} \rightarrow \sqrt{Z_{c}} c^{a}$ & $\zeta_{a} \rightarrow{\sqrt{Z_{c}}}^{-1} \zeta_{a}$ \\
\hline$\left(\psi_{R_{i}}, \overline{\psi_{R_{i}}}\right) \rightarrow \sqrt{Z_{\psi_{R}}}\left(\psi_{R_{i}}, \overline{\psi_{R_{i}}}\right)$ & $\left(R^{i}, \bar{R}^{i}\right) \rightarrow{\sqrt{Z_{\psi_{R}}}}^{-1}\left(R^{i}, \bar{R}^{i}\right)$ \\
\hline$\left(\psi_{L_{i}}, \overline{\psi_{L_{i}}}\right) \rightarrow \quad\left(\psi_{L_{i}}, \overline{\psi_{L_{i}}}\right)$ & \\
\hline$\Phi_{m} \rightarrow \sqrt{Z_{\Phi}} \Phi_{m}$ & $\mathscr{Y}^{m} \rightarrow{\sqrt{Z_{\Phi}}}^{-1} \mathscr{Y}^{m}$ \\
\hline \multicolumn{2}{|c|}{ BRST gauge-fixing fields and parameter } \\
\hline \multicolumn{2}{|c|}{$B^{a}, \bar{c}^{a}, \xi$}$\rightarrow\left\{{\sqrt{Z_{G}}}^{-1} B^{a},{\sqrt{Z_{G}}}^{-1} \bar{c}^{a}, Z_{G} \xi\right\}$ \\
\hline
\end{tabular}

Table 1: The renormalization transformations for coupling constants, dynamical fields, BRST sources, and gauge-fixing fields and parameter. In our model with irreducible representations, and at one-loop level, the renormalization constants $Z_{\varphi}$ remain diagonal in group space.

This renormalization transformation must be applied on the BRST-invariant part of the treelevel action, $S_{0, \text { inv }}$, but not to its part that contains the evanescent component of the fermion kinetic term. Once this transformation is applied, we obtain an invariant counterterm action $S_{\text {ct,inv }}$,

$$
S_{0, \text { inv }} \stackrel{\text { Table }}{\longrightarrow} S_{0, \text { inv }}+S_{\mathrm{ct}, \text { inv }}
$$

invariant in the sense that the Slavnov-Taylor identity holds:

$$
\mathscr{S}_{d}\left(S_{0, \text { inv }}+S_{\mathrm{ct}, \text { inv }}\right)=0 .
$$

However in the BMHV scheme a more general counterterm structure is expected:

$$
S_{\mathrm{sct}}+S_{\mathrm{fct}}=\left(S_{\mathrm{sct}, \text { inv }}+S_{\mathrm{sct}, \text { evan }}\right)+\left(S_{\mathrm{fct}, \text { inv }}+S_{\mathrm{fct}, \text { restore }}+S_{\mathrm{fct}, \text { evan }}\right) .
$$

Here, both the invariant singular $S_{\text {sct,inv }}$ (i.e. involving $1 / \varepsilon$ poles) and finite $S_{\text {fct,inv }}$ counterterms are generated by a renormalization transformation as in Eq. (4.1). There can exist additional, but 
purely evanescent singular counterterms $S_{\text {sct,evan }}$ needed to cancel additional $1 / \varepsilon$ poles from loop diagrams, as we will see below. Most importantly are the finite counterterms $S_{\mathrm{fct}}$,restore necessary to restore the BRST symmetry, and their determination is one of our central goals. Finally there can be additional counterterms $S_{\mathrm{fct} \text {,evan }}$ which are both finite and evanescent. Adding or changing such counterterms can swap between different options as in Eq. (2.5); these counterterms vanish in the 4-dimensional limit, but they can affect calculations at higher orders.

\section{One-loop Singular Counterterms}

The UV-divergences $\left.\Gamma\right|_{\text {div }} ^{(1)}$ of 1PI Green's functions are evaluated at the one-loop $\left(\hbar^{1}\right)$ order, using the diagrammatic method. We performed the calculations in $d=4-2 \varepsilon$ dimensions, with the Mathematica packages FeynArts [27] and FeynCalc [28, 29]; the $\varepsilon$-expansion has been cross-checked using the FeynCalc's interface FeynHelpers [30] to Package-X [31].

Based on dimensionality arguments, it is sufficient to evaluate the one-loop corrections to the self-energies of the fields and to the vertices of the theory. Loop corrections to the vertex terms $K_{\phi} s_{d} \phi$ with BRST sources $K_{\phi}$ have to be computed as well. Their complete list can be found in Section 5 of [1]. From there the one-loop (order $\hbar^{1}$ ) singular counterterm action $S_{\text {sct }}^{(1)}$ is defined such as to cancel the divergent parts of the one-loop vertices, and can be expressed as

$$
S_{\mathrm{sct}}^{(1)}=-\left.\Gamma\right|_{\mathrm{div}} ^{(1)}=S_{\mathrm{sct}, \text { inv }}^{(1)}+S_{\mathrm{sct}, \mathrm{evan}}^{(1)}
$$

where the first term arises from renormalization transformation as in Eq. (4.1) and is given by:

$$
\begin{aligned}
S_{\mathrm{ct}, \text { inv }}= & \frac{\delta Z_{G}}{2} L_{G}+\frac{\delta Z_{\psi_{R}}}{2} \overline{L_{\psi_{R}}}+\frac{\delta Z_{\Phi}}{2} L_{\Phi}+\frac{\delta Z_{c}}{2} L_{c} \\
& +\frac{\delta g}{g} L_{g}+\left(\delta\left(Y_{R}\right)_{i j}^{m} L_{Y_{R i j}}^{m}+\text { h.c. }\right)+\delta \lambda^{m n o p} L_{\lambda^{m n o p}}
\end{aligned}
$$

using " $L_{\varphi}$ functionals" $\left(N_{\varphi}\right.$ denoting the field-counting operator for $\left.\varphi: N_{\varphi}=\int \mathrm{d}^{d} x \varphi_{i}(x) \frac{\delta}{\delta \varphi_{i}(x)}\right)$

$$
\begin{aligned}
L_{G} & =b_{d} \int \mathrm{d}^{d} x\left(\rho_{a}^{\mu}+\partial^{\mu} \bar{c}_{a}\right) G_{\mu}^{a}=\left(N_{G}-N_{\bar{c}}-N_{B}-N_{\rho}+2 \xi \frac{\partial}{\partial \xi}\right) S_{0} \equiv \mathscr{N}_{G} S_{0}, \\
L_{c} & =-b_{d} \int \mathrm{d}^{d} x \zeta_{a} c^{a}=\left(N_{c}-N_{\zeta}\right) S_{0} \equiv \mathscr{N}_{c} S_{0}, \\
L_{\Phi} & =b_{d} \int \mathrm{d}^{d} x \mathscr{Y}^{m} \Phi_{m}=\left(N_{\Phi}-N_{\mathscr{Y}}\right) S_{0} \equiv \mathscr{N}_{\Phi} S_{0}, \\
\overline{L_{\psi_{R}}} & =-b_{d} \int \mathrm{d}^{d} x\left(\bar{R}^{i} \mathbb{P}_{\mathrm{R}} \psi_{i}+\bar{\psi}_{i} \mathbb{P}_{\mathrm{L}} R^{i}\right)-S_{0, \text { evan }}=-\left(N_{\psi}^{R}+N_{\bar{\psi}}^{L}-N_{\bar{R}}-N_{R}\right) S_{0, \text { inv }} \equiv \mathscr{N}_{\psi}^{R} S_{0, \text { inv }},
\end{aligned}
$$

and

$$
L_{g} \equiv g \frac{\partial S_{0}}{\partial g}, \quad L_{Y_{R} i j}^{m} \equiv \frac{\partial S_{0}}{\partial\left(Y_{R}\right)_{i j}^{m}}, \quad L_{\lambda_{\text {mnop }}} \equiv \frac{\partial S_{0}}{\partial \lambda_{\text {mnop }}},
$$

while the second term contains purely evanescent quantities. The renormalization constants $\delta Z_{\varphi}$ agree with the usual ones (see e.g. $[18,19,20]$ ) and read (using the group invariants from [1])

$$
\delta Z_{G}^{(1)}=\frac{\hbar}{16 \pi^{2} \varepsilon} g^{2} \frac{(13-3 \xi) C_{2}(G)-4 S_{2}(R)-S_{2}(S)}{6},
$$




$$
\begin{aligned}
\delta Z_{\psi_{R}}^{(1)}= & \frac{-\hbar}{16 \pi^{2} \varepsilon}\left(g^{2} \xi C_{2}(R)+\frac{Y_{2}(R)}{2}\right), \\
\delta Z_{\Phi}^{(1)}= & \frac{\hbar}{16 \pi^{2} \varepsilon}\left(g^{2}(3-\xi) C_{2}(S)-Y_{2}(S)\right), \\
\delta Z_{c}^{(1)}= & \frac{\hbar}{16 \pi^{2} \varepsilon} g^{2} \frac{(22-6 \xi) C_{2}(G)-4 S_{2}(R)-S_{2}(S)}{6}, \\
\delta g^{(1)} / g= & \frac{-\hbar}{16 \pi^{2} \varepsilon} g^{2} \frac{22 C_{2}(G)-4 S_{2}(R)-S_{2}(S)}{12}, \\
\delta\left(Y_{R}\right)_{i j}^{m,(1)}= & \frac{\hbar}{16 \pi^{2} \varepsilon}\left(\left(Y_{R}^{n}\left(Y_{R}^{m}\right)^{*} Y_{R}^{n}\right)-g^{2} \frac{2 C_{2}(R)(3+\xi)-C_{2}(S)(3-\xi)}{2} Y_{R}^{m}\right)_{i j} \\
& -\left(\delta Z_{\psi_{R}}^{(1)}+\delta Z_{\Phi}^{(1)} / 2\right)\left(Y_{R}\right)_{i j}^{m}, \\
\delta \lambda_{\text {mnop }}^{(1)}= & \frac{\hbar}{16 \pi^{2} \varepsilon} \frac{1}{2}\left(3 g^{4} A-g^{2} \xi \Lambda^{S}-4 H+\Lambda^{2}\right)_{\text {mnop }}-2 \delta Z_{\Phi}^{(1)} \lambda_{\text {mnop }} .
\end{aligned}
$$

The renormalization transformation applied to $S_{0, \text { inv }}$, provides most of the counterterms. As a result the counterterms $S_{\text {sct,inv }}^{(1)}$ contain, from the term $\delta Z_{\psi_{R}} \overline{L_{\psi_{R}}}$, only corrections to the purely 4dimensional right-handed fermion kinetic term: only the right-handed fermion component renormalizes, while the fictitious left-handed component does not.

The evanescent counterterms, specific to the BMHV scheme and appearing in Eq. (4.4), are:

$$
\begin{aligned}
S_{\text {sct,evan }}^{(1)}= & \frac{-\hbar}{16 \pi^{2} \varepsilon}\left\{g^{2} \frac{S_{2}(R)}{3}\left(2\left(\widetilde{S}_{G G}+\widetilde{S}_{G G G}+\widetilde{S}_{G G G G}\right)+\int \mathrm{d}^{d} x \frac{1}{2} \bar{G}^{a \mu} \widehat{\partial}^{2} \bar{G}_{\mu}^{a}\right)\right. \\
& \left.+Y_{2}(S)\left(\left(\widetilde{S}_{\Phi \Phi}+\widetilde{S}_{\Phi G \Phi}+\widetilde{S}_{\Phi G G \Phi}\right)+\frac{2}{3} \widehat{S_{\Phi \Phi}}\right)\right\},
\end{aligned}
$$

using the monomials introduced in Eq. (2.12), with

$$
\widetilde{S}_{\mathscr{O}}=\bar{S}_{\mathscr{O}}-S_{\mathscr{O}} \quad \text { for } \mathscr{O}=G G, G G G, G G G G, \Phi \Phi, \Phi G \Phi, \Phi G G \Phi .
$$

A bar (resp. a hat) over them signify that they are completely projected onto the 4 (resp. the $d-4$ ) dimensional space. The evanescent terms $\widetilde{S}_{\mathscr{O}}$ are still gauge invariant, while the two additional terms present in Eq. (4.14), contributions to the gauge boson and scalar self-energies, are not gauge invariant. This result is also specific to our choice of the regularized, $d$-dimensional theory, based on Eq. (2.7a). For example, replacing the object $\mathbb{P}_{L} \gamma_{\mu} \mathbb{P}_{R}$ by $\gamma_{\mu} \mathbb{P}_{R}$ in the fermion-gauge boson interaction, Eq. (2.5), would have generated a new evanescent counterterm to the fermion selfenergy: $\hbar /\left(16 \pi^{2} \varepsilon\right) g^{2} C_{2}(R) \int_{x} i \bar{\psi}_{i} \widehat{\partial} \mathbb{P}_{\mathrm{R}} \psi_{i}$, as well as many more evanescent contributions to the gauge boson self-energy [1].

\section{BRST Restoration and the Finite BRST-restoring Counterterms}

The $d$-dimensional theory breaks the BRST invariance, both at tree-level and at loop-level, via the singular counterterms $S_{\mathrm{sct}}^{(1)}$ that render the theory finite, see Section 4 . We thus seek at restoring this invariance, central to the consistency of the theory, by determining suitable BRST restoring counterterms $S_{\mathrm{fct}}^{(1)}$ that will ensure the finite renormalized effective action $\Gamma_{\mathrm{Ren}}$ satisfies again the Slavnov-Taylor identity,

$$
\mathscr{S}\left(\Gamma_{\text {Ren }}\right)=0 .
$$


Including all these counterterms, the one-loop $d$-dimensional effective action can be written as

$$
\Gamma_{\mathrm{DReg}}^{(1)}=\Gamma^{(1)}+S_{\mathrm{sct}}^{(1)}+S_{\mathrm{fct}}^{(1)},
$$

where $\Gamma^{(1)}$ denotes the effective action from tree-level and genuine 1-loop diagrams without counterterms. The renormalized one-loop effective action $\Gamma_{\operatorname{Ren}}^{(1)}$ is then obtained in the physical 4dimensional space from $\Gamma_{\mathrm{DReg}}^{(1)}$, by first taking the $d \rightarrow 4$ limit and then, setting all remaining evanescent objects to zero. This operation is denoted by $\operatorname{LIM}_{d \rightarrow 4}$, and we have: $\operatorname{LIM}_{d \rightarrow 4} \Gamma_{\mathrm{DReg}}^{(1)}=\Gamma_{\mathrm{Ren}}^{(1)}$.

In $d$ dimensions at the one-loop level the Slavnov-Taylor identity is expressed by

$$
\mathscr{S}_{d}\left(\Gamma_{\mathrm{DReg}}^{(1)}\right)=\mathscr{S}_{d}\left(\Gamma^{(1)}\right)+b_{d} S_{\mathrm{sct}}^{(1)}+b_{d} S_{\mathrm{fct}}^{(1)} ;
$$

here the $d$-dimensional linearized BRST operator $b_{d}[1,32]$ is defined by:

$$
b_{d}=s_{d}+\int \mathrm{d}^{d} x\left(\frac{\delta S_{0}}{\delta G_{\mu}^{a}} \frac{\delta}{\delta \rho_{a}^{\mu}}+\frac{\delta S_{0}}{\delta c^{a}} \frac{\delta}{\delta \zeta_{a}}+\frac{\delta S_{0}}{\delta \Phi_{m}} \frac{\delta}{\delta \mathscr{Y}^{m}}+\frac{\delta S_{0}}{\delta \psi_{i}} \frac{\delta}{\delta \bar{R}^{i}}+\frac{\delta S_{0}}{\delta \bar{\psi}_{i}} \frac{\delta}{\delta R^{i}}\right)
$$

and terms of higher loop order have been neglected. The finite symmetry-restoring counterterms $S_{\text {fct }}^{(1)}$ are defined such that their $b_{d}$-variation cancels the symmetry breaking. They are also not unique, since they can always be expanded as (see also Eq. (4.3))

$$
S_{\mathrm{fct}}^{(1)}=S_{\mathrm{fct}, \text { inv }}^{(1)}+S_{\mathrm{fct}, \text { restore }}^{(1)}+S_{\mathrm{fct}, \mathrm{evan}}^{(1)} .
$$

Here, $S_{\text {fct,inv }}^{(1)}$ originates from the renormalization transformation (4.1) and is symmetry invariant in the sense of (4.2); the evanescent counterterms $S_{\text {fct,evan }}^{(1)}$ vanish in the $\operatorname{LIM}_{d \rightarrow 4}$ by definition and are therefore irrelevant for symmetry restoration at the one-loop level ${ }^{1}$. Therefore, the actual symmetry-restoring one-loop counterterms are given by $S_{\text {fct,restore }}^{(1)}$.

The regularized quantum action principle for dimensional regularization [11] implies

$$
\mathscr{S}_{d}\left(\Gamma^{(1)}\right)=\widehat{\Delta} \cdot \Gamma^{(1)},
$$

where $\widehat{\Delta}=s_{d} S_{0}$ is the original tree-level BRST symmetry breaking vertex operator Eqs. (3.3) and (3.4), that is inserted in the generating functional of one-loop regularized Green's functions. Thus, the condition that the Slavnov-Taylor identity is satisfied at the one-loop level in the 4dimensional limit Eq. (5.1), can be reformulated as

$$
0=\underset{d \rightarrow 4}{\operatorname{LIM}}\left(\left[\widehat{\Delta} \cdot \Gamma^{(1)}\right]_{\mathrm{div}}+b_{d} S_{\mathrm{sct}}^{(1)}+\left[\widehat{\Delta} \cdot \Gamma^{(1)}\right]_{\mathrm{fin}}+b_{d} S_{\mathrm{fct}, \text { restore }}^{(1)}\right),
$$

where the subscripts "div"/“fin" denote the $1 / \varepsilon$ and finite parts, respectively. This is the defining condition for the one-loop symmetry-restoring counterterms.

The two divergent quantities $\left[\widehat{\Delta} \cdot \Gamma^{(1)}\right]_{\text {div }}$ and $b_{d} S_{\text {sct }}^{(1)}$ must add up to a finite quantity by construction. However, it turns out that they actually cancel each other:

$$
b_{d} S_{\mathrm{sct}}^{(1)}=\frac{-\hbar}{16 \pi^{2} \varepsilon}\left\{g^{2} \frac{\xi C_{2}(G)}{2} \widehat{\Delta}+g^{2} \frac{S_{2}(R)}{3} b_{d} \int \mathrm{d}^{d} x \frac{1}{2} \bar{G}^{a \mu} \widehat{\partial}^{2} \bar{G}_{\mu}^{a}+\frac{2 Y_{2}(S)}{3} b_{d} \widehat{S_{\Phi \Phi}}\right\}=-[\widehat{\Delta} \cdot \Gamma]_{\mathrm{div}}^{(1)} .
$$

\footnotetext{
${ }^{1}$ The choice of one-loop evanescent counterterms will have an impact on two- and higher-loop calculations.
} 
We can now proceed to evaluating the finite quantity $\operatorname{LIM}_{d \rightarrow 4}\left[\widehat{\Delta} \cdot \Gamma^{(1)}\right]_{\text {fin }}$ appearing in Eq. (5.7). This is the central quantity that describes the one-loop symmetry breaking caused by the BMHV scheme, and that will allow us to find the finite BRST-restoring counterterms $S_{\mathrm{fct}, \text {,restore }}^{(1)}$ At first order in $\hbar$, the finite quantity may be expressed as

$$
\underset{d \rightarrow 4}{\operatorname{LIM}}\left[\widehat{\Delta} \cdot \Gamma^{(1)}\right]_{\text {fin }}=\left[N[\widehat{\Delta}] \cdot \Gamma_{\operatorname{Ren}}\right]^{(1)},
$$

$N[\mathscr{O}]$ denotes a renormalized local operator (also called "normal product”), defined as an insertion of $\mathscr{O}$ followed, in the context of Dimensional Regularization and Renormalization (subscript "Ren"), by a minimal subtraction prescription [33] and taking the $\operatorname{LIM}_{d \rightarrow 4}$.

The renormalized insertion of the evanescent breaking $\widehat{\Delta}$ in the effective action: $\left[N[\widehat{\Delta}] \cdot \Gamma_{\operatorname{Ren}}\right]^{(1)}$, can be evaluated using an identity due to Bonneau [34, 35, 36]. A particular simple form of this identity arises at one-loop [1]:

$$
\left[N[\widehat{\Delta}] \cdot \Gamma_{\text {Ren }}\right]^{(1)}=\underset{d \rightarrow 4}{\operatorname{LIM}}\left(- \text { r.s.p. }[\check{\Delta} \cdot \Gamma]_{\check{g}=0}^{(1)}\right) .
$$

On the right-hand side, "r.s.p." means the residue of the simple pole in $v=4-d=2 \varepsilon$ of the 1PI Green's function under consideration, and the operator $\breve{\Delta}$ is obtained from $\widehat{\Delta}$ by formally replacing all its evanescent Lorentz structures by their corresponding $d$-dimensional versions contracted ${ }^{2}$ with the symmetric "metric"-tensor $\breve{g}_{\mu \nu}$, possessing the following properties:

$$
\check{g}_{\mu \nu} g^{v \rho}=\check{g}_{\mu \nu} \hat{g}^{v \rho}=\check{g}_{\mu}^{\rho}, \quad \quad \check{g}_{\mu v} \bar{g}^{v \rho}=0, \quad \check{g}_{\mu}^{\mu}=1 .
$$

We see the significant advantage of the Bonneau identity: $\left[N[\widehat{\Delta}] \cdot \Gamma_{\mathrm{Ren}}\right]^{(1)}$ can be evaluated at fixed loop order by determining a limited finite number of specific UV-divergent 1PI 1-loop diagrams.

We now evaluate the different non-zero contributions, listed in Table 2, entering in $[N[\widehat{\Delta}]$. $\left.\Gamma_{\text {Ren }}\right]^{(1)}$, using Eq. (5.10). They can be expressed as a 4-dimensional linear BRST transformation $b$ of field monomials ( $b$ is the restriction to 4 dimensions of $b_{d}$ Eq. (5.4), based on the 4-dimensional action $S_{0}^{(4 D)}$ instead), therefore allowing us to determine the BRST-restoring finite 1-loop counterterms $S_{\text {fct,restore }}^{(1)}$, defined, from Eq. (5.7), such that $b S_{\text {fct,restore }}^{(1)}$ cancels $\left[N[\widehat{\Delta}] \cdot \Gamma_{\text {Ren }}\right]^{(1)}$. We also obtain the expression for the gauge anomalies. These finite counterterms are sufficient to restore the BRST invariance when the anomaly cancellation condition is met [26].

\begin{tabular}{c|c}
\hline Amplitude in Sect. 6.2 of Ref. [1] & Contribution to $\left[N[\widehat{\Delta}] \cdot \Gamma_{\text {Ren }}\right]^{(1)} \times\left(\frac{\hbar}{16 \pi^{2}}\right)^{-1}$ \\
\hline$\check{\Delta} c^{a} G_{\mu}^{b}$ & $g^{2} \frac{S_{2}(R)}{3} \int \mathrm{d}^{4} x\left(\partial^{\mu} c_{a}\right)\left(\partial^{2} G_{\mu}^{a}\right)$ \\
\hline$\check{\Delta} c^{a} G_{\mu}^{b} G_{v}^{c}$ & $g^{2} \frac{S_{2}(R)}{3} \int \mathrm{d}^{4} x g f^{a b c} c_{a} G_{\mu}^{b}\left(\partial^{2} g^{\mu v}-2 \partial^{\mu} \partial^{v}\right) G_{v}^{c}$ \\
& $-g^{2} \frac{d_{R}^{a b c}}{3} \int \mathrm{d}^{4} x g \varepsilon^{\mu v \rho \sigma} c_{a}\left(\partial_{\rho} G_{\mu}^{b}\right)\left(\partial_{\sigma} G_{v}^{c}\right)$ \\
\hline
\end{tabular}

Table 2: The non-zero contributions to the evaluation of $\left[N[\widehat{\Delta}] \cdot \Gamma_{\text {Ren }}\right]^{(1)}$ at 1-loop. These contributions can be reorganized as $b$-transformations of field monomials.

\footnotetext{
${ }^{2}$ For example: $\widehat{p}^{2}=p_{\mu} p_{v} \hat{g}^{\mu \nu} \rightarrow p_{\mu} p_{v} \check{g}^{\mu v} \equiv \breve{p}^{2}$, and so on...
} 


\begin{tabular}{|c|c|}
\hline Amplitude in Sect. 6.2 of Ref. [1] & Contribution to $\left[N[\widehat{\Delta}] \cdot \Gamma_{\text {Ren }}\right]^{(1)} \times\left(\frac{\hbar}{16 \pi^{2}}\right)^{-1}$ \\
\hline$\breve{\Delta} c^{a} G_{\mu}^{b} G_{V}^{c} G_{\rho}^{d}$ & $\begin{array}{r}g^{4} \frac{\mathscr{A}_{R}^{a b c d}}{6} \int \mathrm{d}^{4} x c_{a} \partial^{v}\left(G_{\mu}^{b} G^{c \mu} G_{v}^{d}\right) \\
-g^{4} \frac{\mathscr{D}_{R}^{a b c d}}{3 \times 3 !} \int \mathrm{d}^{4} x c_{a} \varepsilon^{\mu v \rho \sigma} \partial_{\sigma}\left(G_{\mu}^{b} G_{v}^{c} G_{\rho}^{d}\right)\end{array}$ \\
\hline \multicolumn{2}{|r|}{ 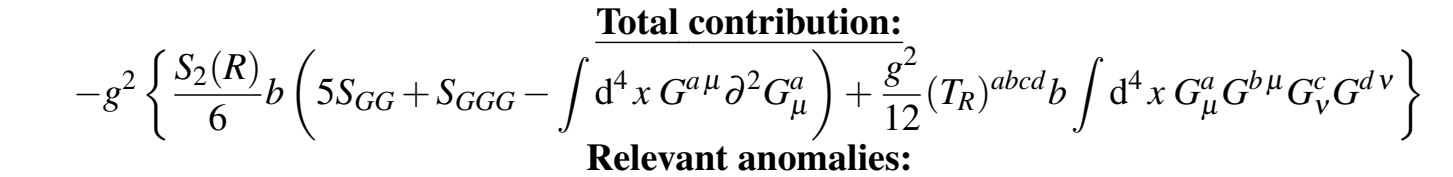 } \\
\hline \multicolumn{2}{|c|}{$-g^{2}\left\{\frac{d_{R}^{a b c}}{3} \int \mathrm{d}^{4} x g \varepsilon^{\mu \nu \rho \sigma} c_{a}\left(\partial_{\rho} G_{\mu}^{b}\right)\left(\partial_{\sigma} G_{v}^{c}\right)+\frac{\mathscr{D}_{R}^{a b c d}}{3 \times 3 !} \int \mathrm{d}^{4} \times g^{2} c_{a} \varepsilon^{\mu v \rho \sigma} \partial_{\sigma}\left(G_{\mu}^{b} G_{v}^{c} G_{\rho}^{d}\right)\right\}$} \\
\hline$\breve{\Delta} c^{a} \Phi^{m} \Phi^{n}$ & Contrib.: $\quad-\frac{Y_{2}(S)}{3} b \overline{S_{\Phi \Phi}}$ \\
\hline$\check{\Delta} c^{a} G_{\mu}^{b} \Phi^{m} \Phi^{n}$ & Contrib.: $\quad \frac{\left(\mathscr{C}_{R}\right)_{m n}^{a b}}{3} b \int \mathrm{d}^{4} x \frac{g^{2}}{2} G_{\mu}^{a} G^{b \mu} \Phi^{m} \Phi^{n}$ \\
\hline$\breve{\Delta} c^{a} \bar{\psi}_{i, \alpha} \psi_{j, \beta}$ & $\begin{array}{r}-g^{3}\left[C_{2}(R)-\frac{C_{2}(G)}{4}+(\xi-1)\left(\frac{C_{2}(R)}{6}-\frac{C_{2}(G)}{4}\right)\right] T_{R i j}^{a} \\
-\frac{g}{2}\left(\left(Y_{R}^{m}\right)^{*} T_{\bar{R}}^{a} Y_{R}^{m}\right)_{i j} \int \mathrm{d}^{4} x c_{a} \partial_{\mu}\left(\bar{\psi}_{i} \gamma^{\mu} \mathbb{P}_{\mathrm{R}} \psi_{j}\right)\end{array}$ \\
\hline$\check{\Delta} c^{a} G_{\mu}^{b} \bar{\psi}_{i, \alpha} \psi_{j, \beta}$ & $-i g^{2} \frac{\xi C_{2}(G)}{4} \int \mathrm{d}^{4} x i g^{2} f^{a b c} T_{R_{i j}}^{c} c_{a} \bar{\psi}_{i} G^{b} \mathbb{P}_{\mathrm{R}} \psi_{j}$ \\
\hline$\check{\Delta} c^{a} \Phi^{m} \bar{\psi}_{i, \alpha}^{C} \psi_{j, \beta}$ & $i g^{2} \frac{\xi C_{2}(G)}{4} \int \mathrm{d}^{4} x \frac{g}{2}\left(Y_{R}\right)_{i j}^{n} \theta_{n m}^{a} c_{a} \Phi^{m} \overline{\psi^{C}}{ }_{i} \mathbb{P}_{\mathrm{R}} \psi_{j}$ \\
\hline$\check{\Delta} c^{a} \Phi^{m} \bar{\psi}_{i, \alpha} \psi_{j, \beta}^{C}$ & $i g^{2} \frac{\xi C_{2}(G)}{4} \int \mathrm{d}^{4} x \frac{g}{2}\left(Y_{R}\right)_{i j}^{n *} \theta_{n m}^{a} c_{a} \Phi^{m} \bar{\psi}_{i} \mathbb{P}_{\mathrm{L}} \psi_{j}^{C}$ \\
\hline$\check{\Delta} c^{a} c^{b} \bar{R}_{i, \alpha} \psi_{j, \beta}$ & $g^{2} \frac{\xi C_{2}(G)}{4} \int \mathrm{d}^{4} x i \frac{g^{2}}{2} f^{a b c} T_{R_{i j}^{c}}^{c} c^{a} c^{b} \bar{R}_{i} \mathbb{P}_{\mathrm{R}} \psi_{j}$ \\
\hline$\check{\Delta} c^{a} c^{b} \bar{\psi}_{i, \alpha} R_{j, \beta}$ & $-g^{2} \frac{\xi C_{2}(G)}{4} \int \mathrm{d}^{4} x i \frac{g^{2}}{2} f^{a b c} T_{R}^{c}{ }_{i j}^{c} c^{a} c^{b} \bar{\psi}_{i} \mathbb{P}_{\mathrm{L}} R_{j}$ \\
\hline
\end{tabular}

Total contribution:

$-g^{2}\left(1+\frac{\xi-1}{6}\right) C_{2}(R) b S_{\bar{\psi} \psi}+\frac{\left(\left(Y_{R}^{m}\right)^{*} T_{\bar{R}}^{a} Y_{R}^{m}\right)_{i j}}{2} b \int \mathrm{d}^{4} x g \bar{\psi}_{i} k^{a} \mathbb{P}_{\mathrm{R}} \psi_{j}+g^{2} \frac{\xi C_{2}(G)}{4} b\left(S_{\bar{R} c \psi_{R}}+S_{R c \overline{\psi_{R}}}\right)$

Table 2: (continued)

In these results the following group factors have been employed:

- the fully symmetric symbol $d_{R}^{a b c}=\operatorname{Tr}\left[T_{R}^{a}\left\{T_{R}^{b}, T_{R}^{c}\right\}\right]$, and the fully antisymmetric symbol ${ }^{3} \mathscr{D}_{R}^{a b c d}=$ $(-i) 3 ! \operatorname{Tr}\left[T_{R}^{a} T_{R}{ }^{[b} T_{R}^{c} T_{R}{ }^{d]}\right]=\frac{1}{2}\left(d_{R}^{a b e} f^{e c d}+d_{R}^{a c e} f^{e d b}+d_{R}^{a d e} f^{e b c}\right)$ for the R-representation, following the notations of Ref. [36];

- the notation $\left(T_{R}\right)^{a_{1} \cdots a_{n}}=\operatorname{Tr}\left[T_{R}{ }^{a_{1}} \cdots T_{R}^{a_{n}}\right]$ for the trace of a product of same group generators $T_{R}{ }^{a}$;

\footnotetext{
${ }^{3}$ We employ the standard indicial notation for the (anti-)symmetrization of tensor indices (or subset thereof): $T^{\left[a_{1} \cdots a_{n}\right]}=\frac{1}{n !} \sum_{\pi} \sigma(\pi) T^{a_{\pi(1)}} \cdots T^{a_{\pi(n)}}$, and $T^{\left\{a_{1} \cdots a_{n}\right\}}=\frac{1}{n !} \sum_{\pi} T^{a_{\pi(1)} \cdots T^{a_{\pi(n)}} .}$
} 
- the group factors

$$
\begin{gathered}
\mathscr{A}_{R}^{a b c d}=\frac{1}{2}\left(\left(T_{R}\right)^{a b c d}+\left(T_{R}\right)^{a d c b}+\left(T_{R}\right)^{a c b d}+\left(T_{R}\right)^{a d b c}\right)-\frac{S_{2}(R)}{2}\left(f^{a b e} f^{c d e}+f^{a c e} f^{b d e}\right), \\
\left(\mathscr{C}_{R}\right)_{m n}^{a b} \equiv \operatorname{Tr}\left[2\left\{T_{R}{ }^{a}, T_{R}{ }^{b}\right\}\left(Y_{R}^{m}\right)^{*} Y_{R}^{n}-T_{R}{ }^{a}\left(Y_{R}^{m}\right)^{*} T_{\bar{R}}{ }^{b} Y_{R}^{n}\right], \text { and }\left(\mathscr{S}_{R}\right)_{m n}^{a b} \equiv\left(\left(\mathscr{C}_{R}\right)_{m n}^{a b}+\left(\mathscr{C}_{R}\right)_{m n}^{b a}+m \leftrightarrow\right. \\
n) / 2, \text { completely symmetric by exchanges } a \leftrightarrow b \text { and } m \leftrightarrow n .
\end{gathered}
$$

All in all, we obtain the following BRST-restoring finite counterterms:

$$
\begin{aligned}
S_{\text {fct,restore }}^{(1)}= & \frac{\hbar}{16 \pi^{2}}\left\{g^{2} \frac{S_{2}(R)}{6}\left(5 S_{G G}+S_{G G G}-\int \mathrm{d}^{4} x G^{a \mu} \partial^{2} G_{\mu}^{a}\right)+\frac{Y_{2}(S)}{3} \overline{S_{\Phi \Phi}}\right. \\
& +g^{2} \frac{\left(T_{R}\right)^{a b c d}}{3} \int \mathrm{d}^{4} x \frac{g^{2}}{4} G_{\mu}^{a} G^{b \mu} G_{v}^{c} G^{d v}-\frac{\left(\mathscr{C}_{R}\right)_{m n}^{a b}}{3} \int \mathrm{d}^{4} x \frac{g^{2}}{2} G_{\mu}^{a} G^{b \mu} \Phi^{m} \Phi^{n} \\
& +g^{2}\left(1+\frac{\xi-1}{6}\right) C_{2}(R) S_{\bar{\psi} \psi}-\frac{\left(\left(Y_{R}^{m}\right)^{*} T_{\bar{R}}{ }^{a} Y_{R}^{m}\right)_{i j}}{2} \int \mathrm{d}^{4} x g \bar{\psi}_{i} G^{a} \mathbb{P}_{\mathrm{R}} \psi_{j} \\
& \left.-g^{2} \frac{\xi C_{2}(G)}{4}\left(S_{\bar{R} c \psi_{R}}+S_{R c \overline{\psi_{R}}}\right)\right\}
\end{aligned}
$$

and the following relevant (non-spurious) anomalies:

$$
-\frac{\hbar g^{2}}{16 \pi^{2}}\left(\frac{d_{R}^{a b c}}{3} \int \mathrm{d}^{4} x g \varepsilon^{\mu v \rho \sigma} c_{a}\left(\partial_{\rho} G_{\mu}^{b}\right)\left(\partial_{\sigma} G_{v}^{c}\right)+\frac{\mathscr{D}_{R}^{a b c d}}{3 \times 3 !} \int \mathrm{d}^{4} x g^{2} c_{a} \varepsilon^{\mu v \rho \sigma} \partial_{\sigma}\left(G_{\mu}^{b} G_{v}^{c} G_{\rho}^{d}\right)\right) .
$$

These counterterms Eq. (5.12) constitute our main result, and are necessary and sufficient to restore the BRST symmetry at 1-loop level in the BMHV scheme when the anomalies Eq. (5.13) are cancelled. While they are purely 4-dimensional and non-evanescent, they are not gauge-invariant! Any other finite BRST-invariant counterterms, or even evanescent ones (that will nonetheless vanish after taking the $\operatorname{LIM}_{d \rightarrow 4}$ ), added to $S_{\text {fct,restore }}^{(1)}$, will not contribute to BRST restoration. They will instead only correspond to a change of renormalization prescription for higher-order calculations.

In order to obtain a consistent renormalizable theory, the gauge anomalies Eq. (5.13) must be cancelled (the "anomaly cancellation condition"). This is done by choosing the fermionic content and the associated group representations in a suitable way so as to cancel separately both $\sum_{R} d_{R}^{a b c}$ (the usual triangle anomaly) and $\sum_{R} \mathscr{D}_{R}^{a b c d}$. Left-handed fermions could help [1], since their generated anomalies have an opposite sign than Eq. (5.13).

\section{Renormalization Group Equations}

There exists two approaches for establishing the renormalization group equations. The first is the familiar one based on the usage of renormalization constants, much like in symmetry-invariant renormalizations. The second one is based on the framework of algebraic renormalization theory. Due to the presence of evanescent operators and finite non-evanescent counterterms needed to restore the BRST symmetry, the formalism with renormalization constants will not straightforwardly lead to the true renormalization group equation, that involves only fields and parameters of the original 4-dimensional tree-level action (see also discussion in Ref. [36]). 


\subsection{Method using Algebraic Renormalization}

It can be shown, in the context of the algebraic renormalization framework [26], that the renormalization group equation corresponds to the expansion of the operator insertion

$$
\mu \frac{\partial \Gamma_{\text {Ren }}}{\partial \mu}=\mathscr{O} \cdot \Gamma_{\text {Ren }}
$$

in a suitable basis of operators of UV dimension 4, ghost number 0, with contracted Lorentz indices but free gauge indices (later contracted with group factors from the associated coefficients). These operators must respect the same symmetries as the functional $\mu \partial \Gamma_{\mathrm{Ren}} / \partial \mu$, namely they should respect the BRST equation, the gauge-fixing condition and the ghost equation [26]. The basis of quantum operators respecting these equations can actually be built from its classical approximation [26] by employing the $L_{\varphi}$ functionals, see Eq. (4.6), that become $b$-invariant in 4 dimensions.

As a result, the following renormalization group equation holds at $\hbar$ ( $\sim$ one-loop) order:

$\mu \frac{\partial \Gamma_{\text {Ren }}}{\partial \mu} \stackrel{\mathscr{O}(\hbar)}{=}\left[-\beta^{(1)} g \frac{\partial}{\partial g}-\left(\beta_{Y}^{m}\right)^{(1)} \frac{\partial}{\partial Y_{i j}^{m}}-\beta_{\lambda}^{(1)}\left(\frac{\partial b c d}{\partial \lambda_{a b c d}}+\gamma_{G} \mathscr{N}_{G}+\gamma_{c} \mathscr{N}_{c}+\gamma_{\Phi} \mathscr{N}_{\Phi}+\gamma_{\psi} \mathscr{N}_{\psi}^{R}\right] S_{0}^{(4 D)}\right.$,

where $S_{0}^{(4 D)}$ is the 4-dimensional restriction of the tree-level action of our model, Eq. (2.13). The RHS of equation (6.2) is an expansion in operators and constitutes a system of renormalization group equations for the $\beta$ functions and the $\gamma$ anomalous dimensions. Therefore, we need to evaluate the left-hand side of this equation, in the form of an operator insertion.

Since the dimensional renormalization scale $\mu$ is not a parameter of the original action, the problem of evaluating $\mu \partial \Gamma_{\operatorname{Ren}} / \partial \mu$ is not trivial, and was solved by Bonneau [34] and generalized by Martin [36]. At one-loop the generic but complicated expression can be reduced [1] to

$$
\mu \frac{\partial \Gamma_{\mathrm{Ren}}}{\partial \mu}=N\left[\text { r.s.p. } \Gamma_{\mathrm{DReg}}^{(1)}\right] \cdot \Gamma_{\mathrm{Ren}},
$$

where "r.s.p. $\Gamma_{\mathrm{DReg}}^{(1)}$ " is the residue of the simple pole in $4-d=2 \varepsilon$ of all the 1PI Green's functions described by $\Gamma_{\mathrm{DReg}}$ at $\hbar$ order: it picks up only the divergent contributions from $\Gamma_{\mathrm{DReg}}^{(1)}$, i.e. only the singular counterterms. The latter are then projected onto 4 dimensions due to the renormalization. Therefore, at $\hbar$ order, the renormalization group equation acquires the simple form

$$
\mu \frac{\partial \Gamma_{\mathrm{Ren}}}{\partial \mu} \equiv-2 \varepsilon S_{\mathrm{sct}}^{(1), 4 D}
$$

where $S_{\text {sct }}^{(1), 4 D}$ is just equal to Eq. (4.4) but projected onto 4 dimensions only, and no evanescent operators appear: at one-loop level only, evanescent contributions do not affect RGEs!

By direct comparison of (6.2) with (6.4) and reading the coefficients associated to each independent field products monomials (i.e. $S_{G G}, S_{\bar{\psi} G \psi_{R}}, S_{\Phi G \Phi}$, etc. that are present in the tree-level action $S_{0}^{(4 D)}$ ), we obtain an overdetermined system of equations that relates linear combinations of $\beta$ functions and $\gamma$ anomalous dimensions, at one-loop level. It results in the following solutions:

$$
\beta=\frac{\hbar}{16 \pi^{2}} g^{2}\left(\frac{-22 C_{2}(G)+4 S_{2}(R)+S_{2}(S)}{6}\right),
$$




$$
\begin{aligned}
\beta_{Y_{i j}}^{m}= & \frac{\hbar}{16 \pi^{2}}\left(2\left(Y_{R}^{n}\left(Y_{R}^{m}\right)^{*} Y_{R}^{n}\right)_{i j}-3 g^{2}\left\{C_{2}(R), Y_{R}^{m}\right\}_{i j}+\left(Y_{R}\right)_{i j}^{m} Y_{2}(S)\right. \\
& \left.+\frac{1}{2}\left(\left(Y_{R}\right)_{i j}^{m} Y_{2}(R)+Y_{2}(\bar{R})\left(Y_{R}\right)_{i j}^{m}\right)\right), \\
\beta_{\lambda a b c d}= & \frac{\hbar}{16 \pi^{2}}\left(3 g^{4} A_{a b c d}-4 H_{a b c d}+\Lambda_{a b c d}^{2}+\Lambda_{a b c d}^{Y}-3 g^{2} \Lambda_{a b c d}^{S}\right), \\
\gamma_{G}= & \frac{\hbar}{16 \pi^{2}} g^{2} \frac{(3 \xi-13) C_{2}(G)+4 S_{2}(R)+S_{2}(S)}{6} \\
\gamma_{\psi}= & \frac{\hbar}{16 \pi^{2}} \frac{2 g^{2} \xi C_{2}(R)+Y_{2}(R)}{2} \\
\gamma_{\Phi}= & \frac{\hbar}{16 \pi^{2}}\left(g^{2}(\xi-3) C_{2}(S)+Y_{2}(S)\right) \\
\gamma_{c}= & \frac{\hbar}{16 \pi^{2}} g^{2} \frac{(6 \xi-22) C_{2}(G)+4 S_{2}(R)+S_{2}(S)}{6} .
\end{aligned}
$$

\subsection{Comparison with the Method of Renormalization Constants}

In the "standard textbook" approach to deriving RGEs in the context of DReg, developed in Ref. [37], the $\beta_{i}$ function corresponding to a non-evanescent renormalized parameter $g_{i}$ (with counterterm $\delta g_{i}$ that depends on the renormalization scale $\mu$ ), is defined as $\beta_{i}(\varepsilon) \equiv \partial g_{i} / \partial \ln \mu$, and is function of the $1 / \varepsilon$ pole of $\delta g_{i}$ only. Similarly, the anomalous dimension $\gamma_{\phi}$ for a non-evanescent field $\phi$ is obtained from the renormalization constant $Z_{\phi}$ associated with an irreducible self-energy Green's function, assuming equal renormalization of the fields, via $\gamma_{\phi}(\varepsilon)=\frac{1}{2} \mu d \ln Z_{\phi} / d \mu$. Proceeding similarly for all fields of the theory, one obtains the generic RGE

$$
\mu \frac{\partial \Gamma_{\mathrm{DReg}}}{\partial \mu}=\left(-\sum_{i} \beta_{i}(\varepsilon) \frac{\partial}{\partial g_{i}}+\sum_{\phi} N_{\phi} \gamma_{\phi}(\varepsilon)\right) \Gamma_{\mathrm{DReg}} .
$$

This equation holds even for $\varepsilon \neq 0$. An important detail is that at this level the $\beta$ and $\gamma$ functions are $\varepsilon$-dependent and have the structure

$$
\beta_{i}(\varepsilon), \gamma_{\phi}(\varepsilon)=\mathscr{O}(\varepsilon) \times(\text { tree-level })+\mathscr{O}\left(\varepsilon^{0}\right) \times(\geq 1 \text {-loop level }) .
$$

In principle, all of these remarks apply to the BMHV scheme. However, in this scheme, the action contains evanescent divergent counterterms, see Eq. (4.14), which have no tree-level counterpart. In order to apply the method in the BMHV context, we can amend the tree-level action with additional tree-level "evanescent" parameters $\hat{g}_{k}$ for each term in $S_{\mathrm{sct}, \text { evan }}^{(1)}$, possessing the renormalization transformation $\hat{g}_{k} \rightarrow \hat{g}_{k}+\delta \hat{g}_{k}$. This leads to an RGE with the generic structure

$$
\mu \frac{\partial \Gamma_{\mathrm{DReg}}}{\partial \mu}=\left(-\sum_{k} \beta_{i}(\varepsilon) \frac{\partial}{\partial g_{i}}-\sum_{k} \hat{\beta}_{k}(\varepsilon) \frac{\partial}{\partial \hat{g}_{k}}+\sum_{\phi} N_{\phi} \gamma_{\phi}(\varepsilon)\right) \Gamma_{\mathrm{DReg}}
$$

where the second sum on the right-hand side is over all the new parameters $\hat{g}_{k}$ of the additional evanescent action. These additional parameters have been also considered e.g. in the context of a non-gauge theory, in Ref. [38], and their influence on the RGE discussed. We thus see that our original formulation of the theory in Section 2 corresponds to setting the evanescent parameters $\hat{g}_{k}=0$ at tree-level, for one particular renormalization scale $\mu$ only. Hence, applying the $\operatorname{LIM}_{d \rightarrow 4}$ 
operation at the 1-loop level in the generic RGE (6.14), leads to: $(i)$ all the $\beta_{i}(\varepsilon), \gamma_{\phi}(\varepsilon)$, and $\hat{\beta}_{k}(\varepsilon)$ being quantities of 1-loop order, denoted by $\beta_{i}(0) \equiv \beta_{i}, \gamma_{\phi}(0) \equiv \gamma_{\phi}$ in the $\varepsilon \rightarrow 0$ limit; (ii) the independence in $\hat{g}_{k}$ of $\beta_{i}$ and $\gamma_{\phi}$.

From these comments, applying the $\operatorname{LIM}_{d \rightarrow 4}$ to Eq. (6.14) at the 1-loop level leads to the RGE

$$
\mu \frac{\partial \Gamma_{\text {Ren }}}{\partial \mu}=\left(-\sum_{i} \beta_{i} \frac{\partial}{\partial g_{i}}+\sum_{\phi} N_{\phi} \gamma_{\phi}\right) \Gamma_{\text {Ren }},
$$

where both sides are understood to be evaluated up to 1-loop level. The dependence on $\hat{g}_{k}$ has dropped out, and the non-evanescent coefficients $\beta_{i}, \gamma_{\phi}$ may be evaluated by setting $\hat{g}_{k}=0$. This shows that the correct one-loop RGEs in the BMHV scheme may be obtained by just applying the usual procedure of Refs. [37, 19] from the divergences of the standard non-evanescent renormalization constants, and ignoring the additional evanescent objects contained in the amended tree-level action. We obtain this way [1] the $\beta, \beta_{Y}{ }_{i j}^{m}$ and $\beta_{\lambda_{a b c d}}$ functions from the couplings counterterms, respectively $\delta g^{(1)} / g, \delta\left(Y_{R}\right)_{i j}^{m,(1)}$ and $\delta \lambda_{\text {mnop }}^{(1)}$, and we obtain as well the anomalous dimensions $\gamma_{G}$, $\gamma_{\psi}, \gamma_{\Phi}$ and $\gamma_{c}$, respectively from the field-strength renormalizations $\delta Z_{G}^{(1)}, \delta Z_{\psi_{L}}^{(1)}, \delta Z_{\Phi}^{(1)}$ and $\delta Z_{c}^{(1)}$, and they are exactly the same as those obtained in Section 6.1. However this situation will change at two-loop and higher orders, where e.g. the term $\propto \hat{\beta}_{k}$ is expected to provide finite contributions, and the $\beta_{i}, \gamma_{\phi}$ coefficients might depend on the evanescent parameters $\hat{g}_{k}$.

\section{Conclusion}

In our work [1] we started a systematic study of the BMHV scheme for $\gamma_{5}$ and its application to chiral gauge theories, exemplified at the one-loop level on a theory containing massless right-chiral fermions and scalars, for simplicity restricted to a simple gauge group and irreducible representations. We have focused on the BMHV-specific aspects of renormalization and counterterms.

Extending this model to $d$ dimensions inevitably introduces a freedom of choice for the fermiongauge interaction term. Also, the BRST invariance of the model is broken at tree-level, due to the evanescent part of the fermion kinetic term. Compared to the case of symmetry-invariant renormalizations, the counterterm structure is enlarged, due to this BRST breaking, with new BMHVspecific structures. New counterterms, evanescent yet singular, appear and are necessary at higher orders to ensure unitarity and finiteness, but most of these are still BRST invariant.

Furthermore, since the BRST symmetry is broken at tree-level, Slavnov-Taylor identities are violated at one-(and higher) loop level, and suitable finite symmetry-restoring counterterms need to be found. Our calculation is based on the regularized quantum action principle and the Bonneau identities (this combination of tools has also been used in Ref. [36]).

Finally, the renormalization group equations for the right-handed chiral model have been derived in the context of the BMHV scheme using the algebraic method, and have been compared with those obtained with renormalization factors (inspired by symmetry-invariant regularizations). In case the second method is used, the $\beta$-functions for evanescent couplings should be considered as well, but their effects are inexistent at one-loop level only, and both methods agree. However these evanescent couplings would be of utmost importance for higher loop order calculations. 


\section{Acknowledgments}

H.Bélusca-Maïto thanks the organizers of the Corfu Summer Institute 2019 for the enjoyable environment and activities proposed during the conference, and also acknowledges the financial support conference grant from the COST Action CA16201. A.Ilakovac highly acknowledges the financial support of the Croatian Science Foundation (HRZZ) project HRZZ-IP-2013-11-8799. This work is supported by the HRZZ under the project "PRECIOUS" HRZZ-IP-2016-06-7460.

\section{References}

[1] H. Bélusca-Maïto, A. Ilakovac, M. Mađor-Božinović and D. Stöckinger, Dimensional Regularization and Breitenlohner-Maison / 't Hooft-Veltman Scheme for $\gamma_{5}$ applied to Chiral YM Theories: Full One-Loop Counterterm and RGE Structure, JHEP (2020) accepted, to appear, [2 004.14398 ].

[2] G. Cicuta and E. Montaldi, Analytic renormalization via continuous space dimension, Lett. Nuovo Cim. 4 (1972) 329-332.

[3] C. Bollini and J. Giambiagi, Dimensional Renormalization: The Number of Dimensions as a Regularizing Parameter, Nuovo Cim. B 12 (1972) 20-26.

[4] J. Ashmore, A Method of Gauge Invariant Regularization, Lett. Nuovo Cim. 4 (1972) 289-290.

[5] G. 't Hooft and M. J. G. Veltman, Regularization and Renormalization of Gauge Fields, Nucl. Phys. B44 (1972) 189-213.

[6] J. C. Collins, Renormalization, vol. 26 of Cambridge Monographs on Mathematical Physics. Cambridge University Press, Cambridge, 1986, 10.1017/CBO9780511622656.

[7] D. Akyeampong and R. Delbourgo, Dimensional regularization, abnormal amplitudes and anomalies, Nuovo Cim. A 17 (1973) 578-586.

[8] D. Akyeampong and R. Delbourgo, Dimensional regularization and PCAC, Nuovo Cim. A 18 (1973) 94-104.

[9] D. Akyeampong and R. Delbourgo, Anomalies via dimensional regularization, Nuovo Cim. A 19 (1974) 219-224.

[10] P. Breitenlohner and D. Maison, Dimensional Renormalization of Massless Yang-Mills Theories, 1975.

[11] P. Breitenlohner and D. Maison, Dimensional Renormalization and the Action Principle, Commun. Math. Phys. 52 (1977) 11-38.

[12] P. Breitenlohner and D. Maison, Dimensionally Renormalized Green's Functions for Theories with Massless Particles. 1., Commun. Math. Phys. 52 (1977) 39.

[13] P. Breitenlohner and D. Maison, Dimensionally Renormalized Green's Functions for Theories with Massless Particles. 2., Commun. Math. Phys. 52 (1977) 55.

[14] G. Bonneau, Consistency in Dimensional Regularization With $\gamma_{5}$, Phys. Lett. 96B (1980) 147-150.

[15] S. L. Adler, Axial vector vertex in spinor electrodynamics, Phys. Rev. 177 (1969) 2426-2438.

[16] J. S. Bell and R. Jackiw, A PCAC puzzle: $\pi^{0} \rightarrow \gamma \gamma$ in the $\sigma$ model, Nuovo Cim. A60 (1969) 47-61.

[17] S. L. Adler and W. A. Bardeen, Absence of higher order corrections in the anomalous axial vector divergence equation, Phys. Rev. 182 (1969) 1517-1536. 
[18] M. E. Machacek and M. T. Vaughn, Two Loop Renormalization Group Equations in a General Quantum Field Theory. 1. Wave Function Renormalization, Nucl. Phys. B222 (1983) 83-103.

[19] M. E. Machacek and M. T. Vaughn, Two Loop Renormalization Group Equations in a General Quantum Field Theory. 2. Yukawa Couplings, Nucl. Phys. B236 (1984) 221-232.

[20] M. E. Machacek and M. T. Vaughn, Two Loop Renormalization Group Equations in a General Quantum Field Theory. 3. Scalar Quartic Couplings, Nucl. Phys. B249 (1985) 70-92.

[21] D. Stöckinger, Regularization by dimensional reduction: consistency, quantum action principle, and supersymmetry, JHEP 03 (2005) 076, [hep-ph / 0503129 ].

[22] K. Hieda, A. Kasai, H. Makino and H. Suzuki, 4D $\mathscr{N}=1$ SYM supercurrent in terms of the gradient flow, PTEP 2017 (2017) 063B03, [1703.04802].

[23] H. K. Dreiner, H. E. Haber and S. P. Martin, Two-component spinor techniques and Feynman rules for quantum field theory and supersymmetry, Phys. Rept. 494 (2010) 1-196, [0812 . 1594].

[24] C. Becchi, A. Rouet and R. Stora, Renormalization of Gauge Theories, Annals Phys. 98 (1976) 287-321.

[25] I. V. Tyutin, Gauge Invariance in Field Theory and Statistical Physics in Operator Formalism, 0812.0580 .

[26] O. Piguet and S. P. Sorella, Algebraic renormalization: Perturbative renormalization, symmetries and anomalies, Lect. Notes Phys. Monogr. 28 (1995) 1-134.

[27] T. Hahn, Generating Feynman diagrams and amplitudes with FeynArts 3, Comput. Phys. Commun. 140 (2001) 418-431, [hep-ph/ 0012260$].$

[28] R. Mertig, M. Bohm and A. Denner, FEYN CALC: Computer algebraic calculation of Feynman amplitudes, Comput. Phys. Commun. 64 (1991) 345-359.

[29] V. Shtabovenko, R. Mertig and F. Orellana, New Developments in FeynCalc 9.0, Comput. Phys. Commun. 207 (2016) 432-444, [1601.01167].

[30] V. Shtabovenko, FeynHelpers: Connecting FeynCalc to FIRE and Package-X, Comput. Phys. Commun. 218 (2017) 48-65, [1611.06793].

[31] H. H. Patel, Package-X 2.0: A Mathematica package for the analytic calculation of one-loop integrals, Comput. Phys. Commun. 218 (2017) 66-70, [1612 . 00009 ].

[32] O. Piguet and A. Rouet, Symmetries in Perturbative Quantum Field Theory, Phys. Rept. 76 (1981) 1.

[33] J. C. Collins, Normal Products in Dimensional Regularization, Nucl. Phys. B92 (1975) 477-506.

[34] G. Bonneau, Zimmermann Identities And Renormalization Group Equation In Dimensional Renormalization, Nucl. Phys. B167 (1980) 261-284.

[35] G. Bonneau, Trace and Axial Anomalies in Dimensional Renormalization Through Zimmermann Like Identities, Nucl. Phys. B171 (1980) 477-508.

[36] C. P. Martin and D. Sanchez-Ruiz, Action principles, restoration of BRS symmetry and the renormalization group equation for chiral non-Abelian gauge theories in dimensional renormalization with a non-anticommuting $\gamma_{5}$, Nucl. Phys. B572 (2000) 387-477, [hep-th/9905076].

[37] G. ’t Hooft, Dimensional regularization and the renormalization group, Nucl. Phys. B61 (1973) 455-468.

[38] C. Schubert, The Yukawa Model as an Example for Dimensional Renormalization With $\gamma_{5}$, Nucl. Phys. B323 (1989) 478-492. 